EPJ Web of Conferences 52, 06003 (2013)

DOI: $10.1051 /$ epjconf/20135206003

(C) Owned by the authors, published by EDP Sciences, 2013

\title{
Energy spectrum of UHECRs measured by newly constructed fluorescence detectors in Telescope Array experiment
}

\author{
Toshihiro Fujii ${ }^{1, a}$ for the Telescope Array Collaboration \\ ${ }^{1}$ Institute for Cosmic Ray Research, University of Tokyo, Kashiwa, Chiba 277-8582, Japan
}

\begin{abstract}
Telescope Array (TA) experiment is the largest hybrid detector to observe ultra-high energy cosmic rays (UHECRs) in the northern hemisphere. In the TA experiment, we newly designed and constructed 24 fluorescence detectors (FDs) located at two stations. We report the energy spectrum of UHECRs with energies above $10^{17.5} \mathrm{eV}$ from analyzing data collected by the new FDs during the first 3.7 years in monocular mode.
\end{abstract}

\section{Telescope Array experiment}

Origins of cosmic rays are still unknown during a century. Telescope Array (TA) experiment is the largest hybrid detector toward understanding origins of ultra-high energy cosmic rays (UHECRs) [1]. TA consists of 507 surface detectors (SDs) deployed with $1.2 \mathrm{~km}$ spacing for covering with an effective area of about $700 \mathrm{~km}^{2}$ and of three stations of fluorescence detectors (FDs) looking inward over the SD array, as shown in Fig. 1. A full operation of TA has been started in March 2008.

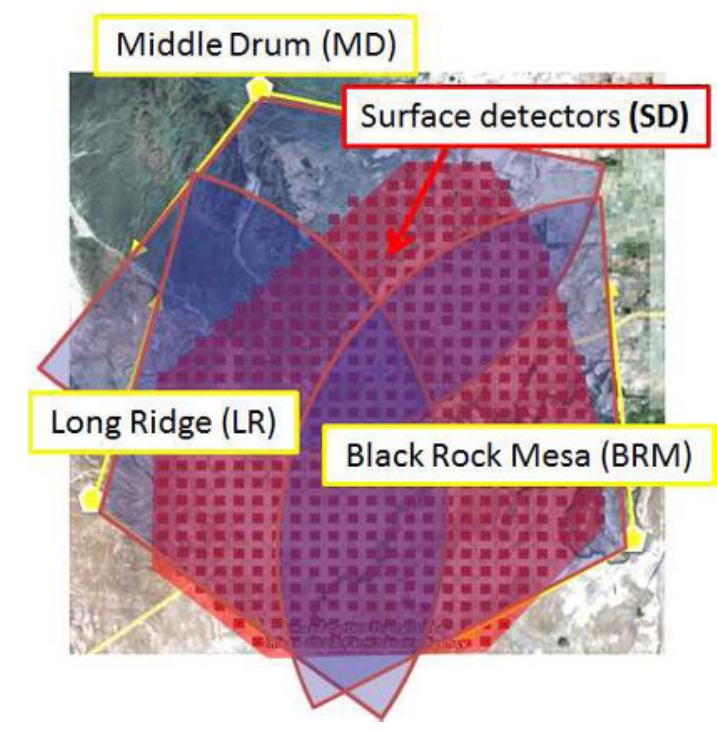

Figure 1. The overview of Telescope Array experiment. The yellow pentagon shows locations of the FD station. The red square shows locations of the surface detector.

One of the stations located at north-west of the array has 14 FDs transported from HiRes experiment[2], so called the Middle Drum station (MD), and the other

\footnotetext{
a e-mail: fujii@icrr.u-tokyo.ac.jp
}

24 FDs located at two stations are newly designed and constructed for the TA experiment, named as the Black Rock Mesa (BRM) and the Long Ridge (LR) station, with new calibrations and atmospheric monitors[3][4][5][6][7]. FD observations detecting atmospheric fluorescence photons emitted by molecules excited by extensive air showers (EAS) provides simulation-independent determinations of primary energies, because productions and energy losses of electro-magnetic components of EASs which are dominant components contributing fluorescence photon emissions are less dependent on hadron interaction models.

\section{Data Set and Reconstruction Procedure}

We explain a data set and an analysis procedure of the FDs in monocular mode which is the method to reconstruct primary cosmic ray information from data measured by only one FD station. Using this monocular analysis, we report the broad energy spectrum of UHECRs above $10^{17.5} \mathrm{eV}$ observed by the newly constructed FDs.

Fig. 2 shows the observation time and data set of FDs located at the BRM and the LR station. Here, we analyze data collected from January 2008 to September 2011 corresponding to 3.7 years.

Since air shower geometries of monocular mode is obtained from injected signal timings of a single FD station, the geometrical resolution is worse than other analysis, such as stereo[8] or hybrid analysis[9]. To reconstruct the geometry of EASs, timing values of each PMTs with signals, $t_{i}$, are fitted by

$$
t_{i}=t_{\text {core }}+\frac{1}{c} \frac{\sin \Psi-\sin \alpha_{i}}{\sin \left(\Psi+\alpha_{i}\right)} .
$$

When the geometry of EASs is determined, a longitudinal development of EAS is estimated by an inverse Monte Carlo method[10]. The inverse Monte Carlo is the way to search an optimum solution of the shower development using Monte Carlo (MC) simulations with changing longitudinal development parameters. 


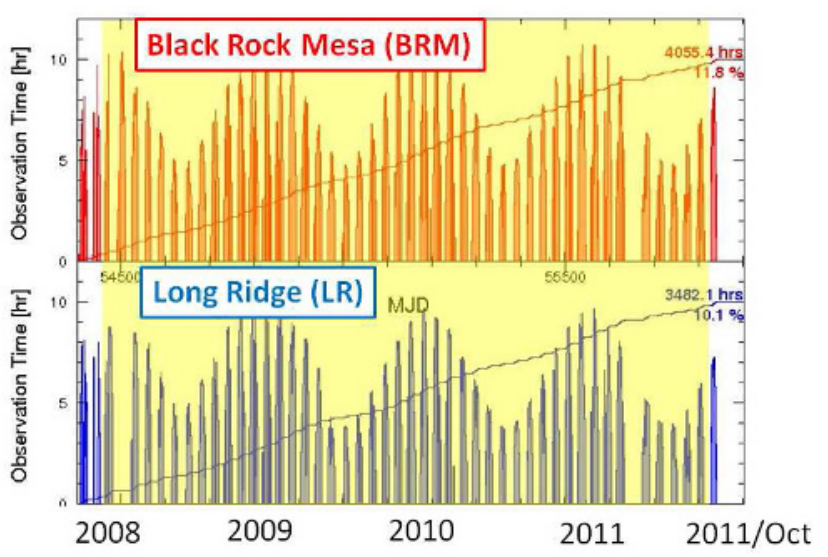

Figure 2. Daily observation hours and its integrated values at BRM and LR station, respectively.

Moreover, the following quality cuts are applied in the monocular analysis because faint shower signals with short tracks are difficult to reconstruct to determine their geometries. Thus, short tracks and small time extent events were removed under following quality cuts.

- The geometrical and the longitudinal fittings are converged.

- The number of selected PMTs is larger than 10.

- The track length is larger than $10^{\circ}$.

- The time extent is larger than $2 \mu \mathrm{s}$.

- The reduced $\chi^{2}$ for the geometrical fitting is less than 20.

- The maximum depth of EAS is observed inside the field of view (FOV) of FD, $X_{\text {start }}<X_{\max }<X_{\text {end }}$ where $X_{\text {start }}$ is the first slant depth where emitted photons are measured by FD, $X_{\text {end }}$ is the last one.

- The reconstructed zenith angle of the EAS is less than $55^{\circ}$.

- The reconstructed core location is within a circle with $25 \mathrm{~km}$ radius from the location of CLF.

\section{Performance of TA FD}

Using the reconstruction methods, we evaluate resolutions of the monocular analysis by MC simulated events before analyses of observed data. At first, we generate artificial data calculated by MC simulations of primary protons generated by QGSJet-II-03 model of CORSIKA simulation[11]. Secondly, we reconstructed this simulated data in monocular analysis, and finally compare reconstructed results with true ones.

The distributions of the difference between simulated and reconstructed values on arrival directions and primary energies of EASs are shown in Fig. 3. The resolution of arrival direction in monocular mode is 7.4 degree as defined within $68 \%$ region. Because of such low geometrical resolution, energy estimation of monocular analysis has a systematic bias. Thus, we corrected this bias which is $+3 \%$ at
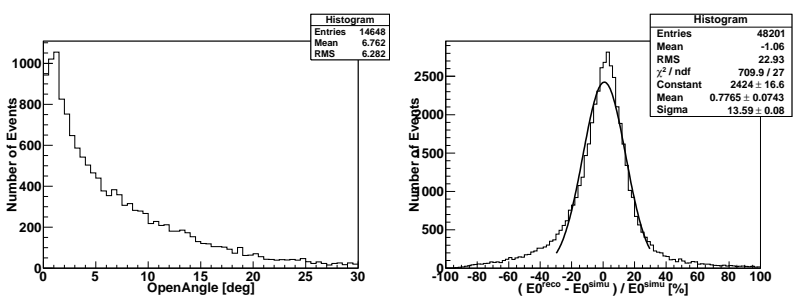

Figure 3. The resolutions of arrival direction and energy of EASs evaluated from MC simulations in monocular mode.

$10^{18.5} \mathrm{eV}$ and $+7 \%$ at $10^{19.5} \mathrm{eV}$ with a energy dependence. As the result, a root-mean-square of the distribution of the histogram is $23 \%$.

To evaluate the energy spectrum of UHECRs, it is essential to calculate an aperture of FD. The aperture can not calculate a simple geometrical factor because it depends on not only the energies, but also the performance of FD, atmospheric models, PMT gains and primary shower species. Thus, we estimate the aperture of FD using MC simulations including these dependences. The aperture of $\mathrm{FD}, A \Omega$, is calculated from a ratio between the number of reconstructed events with the quality cuts and the number of thrown ones[2].

$$
A \Omega(E)=A \Omega^{\mathrm{G}} \cdot \frac{N_{\text {reco }}(E)}{N_{\text {thrown }}(E)},
$$

where $A \Omega^{\mathrm{G}}$ is the thrown aperture region of MC simulation, $N_{\text {reco }}$ is the number of reconstructed events, $N_{\text {thrown }}$ is the number of thrown events.

Since TA was designed stereo observation mode for showers above $10^{19.0} \mathrm{eV}$, we define the combined aperture of the BRM and the LR stations in each monocular mode. When a energetic shower is reconstructed by the both stations, we select one result with a larger number of photoelectrons than another station to avoid the double counting of high energy showers. Using these reconstructed events by both the BRM and the LR combined mode, we estimate the combined aperture of BRM and LR stations with primary protons and irons of the QGSJet-II hadron interaction model as shown in Fig. 4.

In the low energy region less than $10^{18.5} \mathrm{eV}$, the aperture is dependent on the primary species. In contrast, in the high energy region above $10^{19.5} \mathrm{eV}$, it is independent of them.

\section{Data Analysis}

Analyzing the observed data during by the same analysis processes, we obtained histograms of reconstructed energies as shown in Fig 5. There are three types of histograms, BRM (red), LR (blue) and combined (green) energies, respectively.

As further enhancement of our reliability in our analysis, the distributions of the several parameters obtained from the reconstructions of the observed data are compared with the expected ones estimated from MC simulations. A couple of parameters for data and MC comparisons are shown in Fig. 6. In these figures, the black 


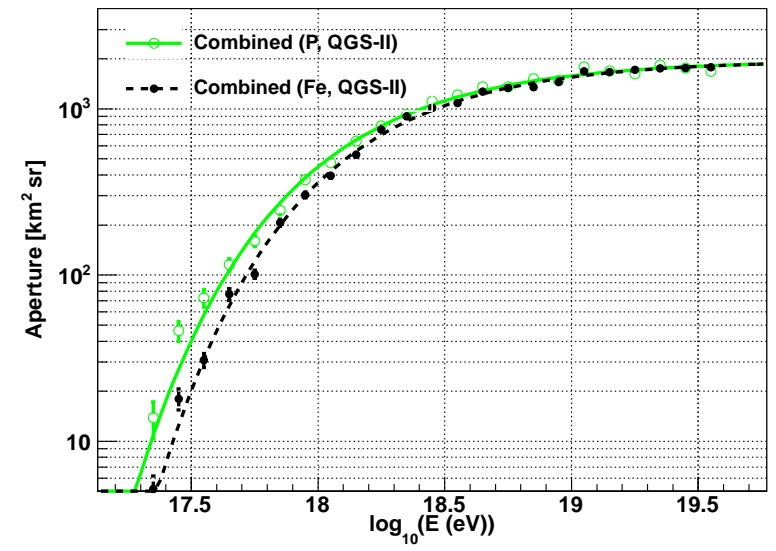

Figure 4. The combined aperture of BRM and LR stations evaluated by MC simulations for primary protons (green solid-line) and irons (black dotted line) in monocular mode.

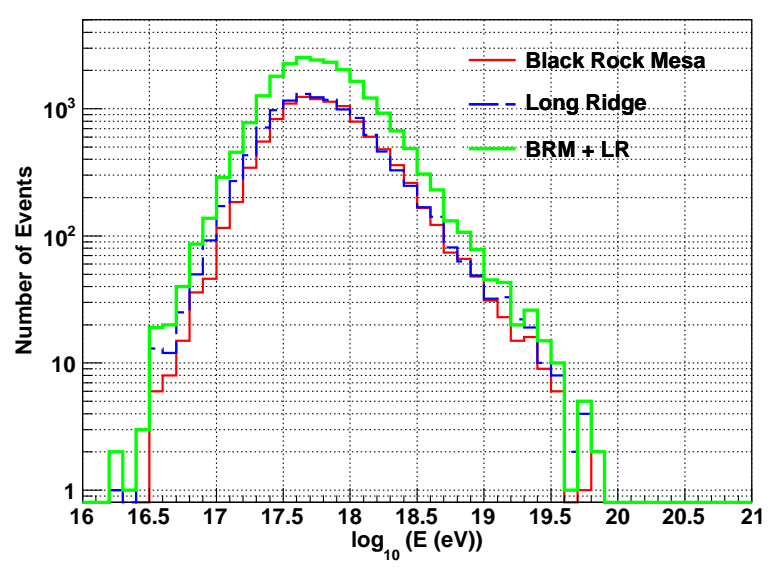

Figure 5. Histograms of reconstructed energies in monocular analysis. The BRM, LR and combined ones are indicated as red, blue and green lines, respectively.

plots indicate the distributions observed at the BRM or the LR stations, the red or blue histograms indicates the expected distributions estimated from MC simulations at the BRM or the LR station for primary protons and irons. The distributions of each MC is normalized to the number of observed events. These plots are in good agreements between data and MC simulations.

Air showers reconstructed by both stations in monocular mode are useful to confirm an accuracy of reconstructed energies independently for Monte Carlo simulations. Assuming both FD stations have the same resolution, $\sigma$, a root-mean-square of distributions of difference of reconstructed energies at the BRM and the LR stations are equivalent to $\sqrt{2} \sigma$. Thus, we estimate the accuracy of our FD monocular analysis from distributions of difference of reconstructed energies at the BRM and the LR stations. In this data set, we found 234 events reconstructed by both stations in monocular mode, and a distribution of
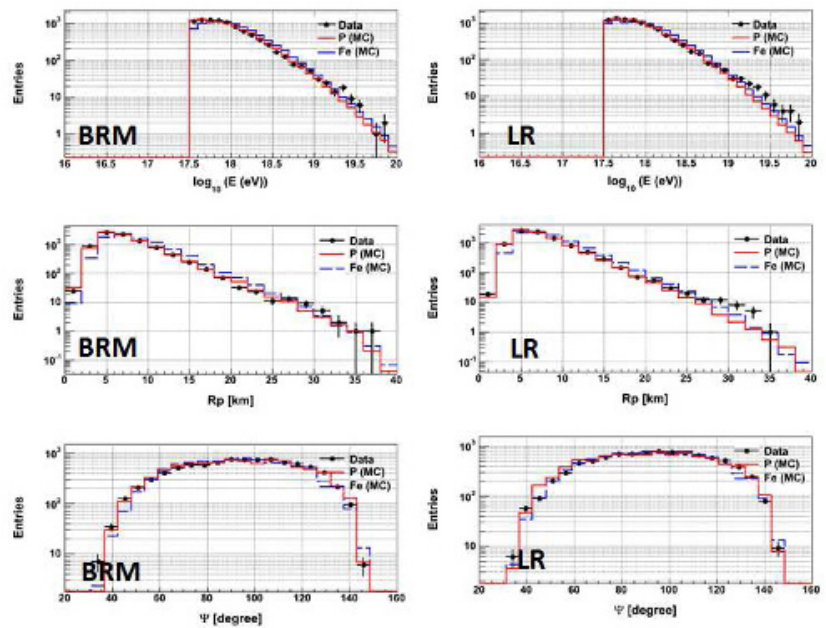

Figure 6. The distributions of energies, impact parameters and angles in a shower detector plane between observed data (plot) and $\mathrm{MC}$ simulations for primary protons (red) and irons (blue) at the BRM (left column) and the LR stations (right column).

these difference is shown in Fig. 7. Converted to a linear value based on Fig. 7, the resolution of FD is estimated as $40 \%$.

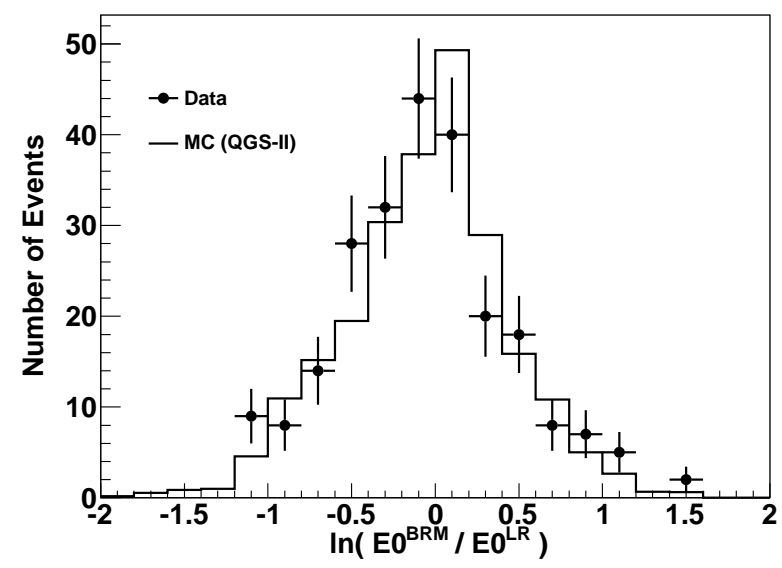

Figure 7. Distributions of difference of reconstructed energies at the BRM and the LR stations. The peak energy of reconstructed 234 showers is $10^{18.6} \mathrm{eV}$. The plot is observed data, the histogram is estimated from our MC simulation.

Finally, we calculate the energy spectrum based on the apertures, the live times and the number of reconstructed showers, as discussed previously. An exposure of FD is giving from the apertures and the live times by

$$
\omega(E)=A \Omega(E) \cdot t
$$

where $t$ indicates the live time and aperture, $A \Omega(E)$, is already estimated in Fig. 4. Moreover, a flux is evaluated by

$$
J(E)=\frac{N(E)}{\omega(E) \cdot \Delta E} .
$$




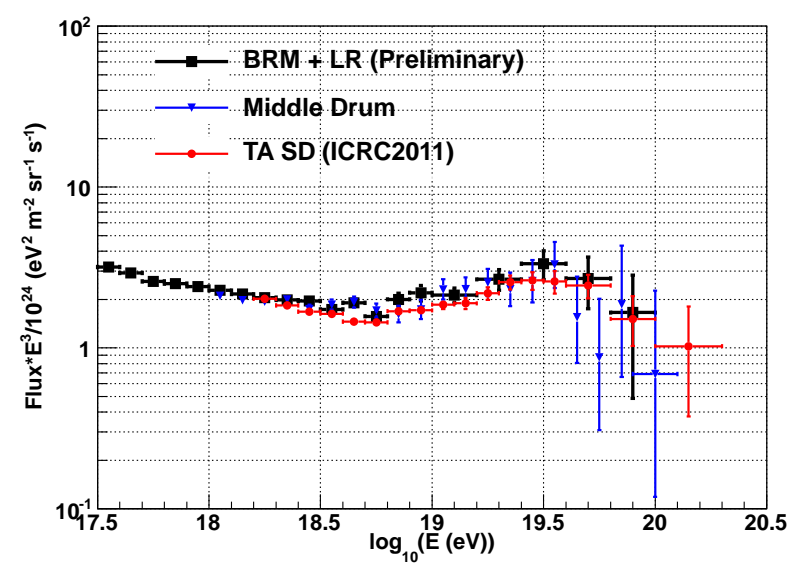

Figure 8. Energy spectra in the TA measurement. The blue plots show the preliminary spectrum analyzing data collected at the BRM and the LR stations, the open squares show the spectrum measured at the Middle Drum station [12], the pink triangles indicate the spectrum measured by TA SD with $27 \%$ scaled as the same energies with TA FD energies [13].

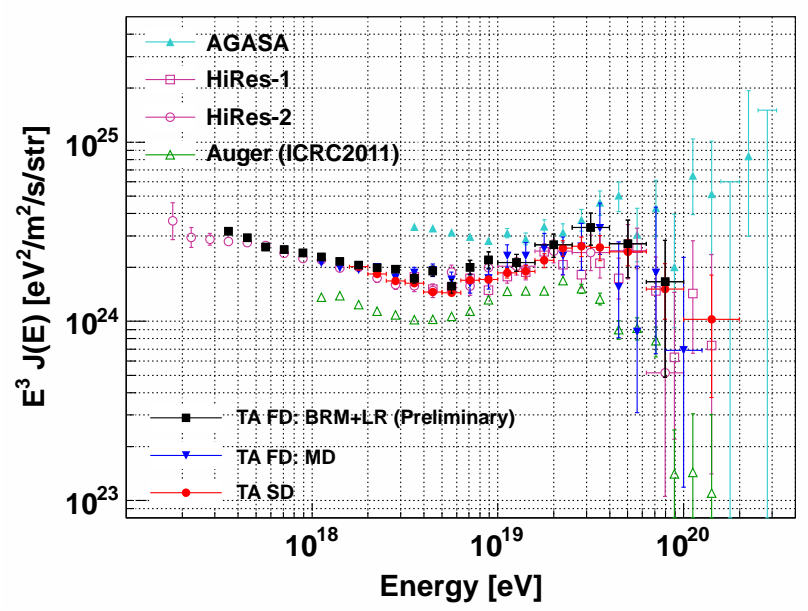

Figure 9. Comparisons of TA spectra with other ones measured by Akeno, AGASA, HiRes and Auger[14][15][2][16].

The calculated preliminary spectrum is shown in Fig. 8 with spectra measured by the TA surface detector and by the FDs at the TA Middle Drum station transported from HiRes experiment. The black plots show the preliminary spectrum analyzing data collected at the BRM and the LR stations, the open squares show the spectrum measured at the Middle Drum station [12], the pink triangles indicate the spectrum measured by TA SD with $27 \%$ scaled as the same energies with TA FD energies [13]. These spectra are in agreement well with each others. Moreover, we compare the observed spectra with other spectra measured by Akeno, AGASA, HiRes and Auger[14][15][2][16]. To see Fig 9, our observed spectra is consistent with the HiRes measurements.

\section{Conclusions}

The newly constructed fluorescence detectors for Telescope Array experiment have a steady observation of UHECRs from 2008. Analyzing the data collected during 3.7 years by the monocular analysis located at the BRM and the LR station, we estimate the energy spectrum with a broad energies above $10^{17.5} \mathrm{eV}$. The obtained energy spectrum is consistent with spectra measured by the TA surface detectors, the Middle Drum FD and the HiRes experiment.

\section{Acknowledgments}

The Telescope Array experiment is supported by the Ministry of Education, Culture, Sports, Science and Technology-Japan, Grant-in-Aid for Scientific Research on Priority Areas, "The Origin of the Highest Energy Cosmic Rays", 2003, Grant-in-Aid for Specially Promoted Research, "Extreme Phenomena in the Universe Explored by Highest Energy Cosmic Rays", 2009 and for Scientific Research (B),20340057, 2008. I gratefully acknowledge the valuable financial support by the Japan Society for the Promotion of Science (JSPS). This experiment is also supported by the U.S. National Science Foundation (NSF) through awards PHY-0307098 and PHY-0601915 (University of Utah) and PHY-0305516 (Rutgers University). The Dr. Ezekiel R. and Edna Wattis Dumke Foundation, The Willard L. Eccles Foundation and The George S. and Dolores Dore Eccles Foundation all helped with generous donations. The State of Utah supported the project through its Economic Development Board, and the University of Utah supported us through the Office of the Vice President for Research. The use of the experimental site became possible by the cooperation of the State of Utah School and Institutional Trust Lands Administration(SITLA), the federal Bureau of Land Management(BLM) and the United States Air Force. We also wish to thank the people and the officials of Millard County, Utah, for their steadfast and warm supports. We gratefully acknowledge the contributions from the technical staffs of our home institutions.

\section{References}

[1] The Telescope Array Project Design Report (2000).

[2] R.U. Abbasi et al., Phys. Rev. Lett., 100(10), 101101 (2008). R.U. Abbasi et al., Phys. Rev. Lett., 104, 161101 (2010).

[3] H. Tokuno et al., Nucl. Instr. and Meth. A, 601, 364 (2009).

[4] Y. Tameda et al., Nucl. Instr. and Meth. A, 609, 227 (2009).

[5] T. Tomida et al., Nucl. Instr. and Meth. A, 654, 653 (2011).

[6] H. Tokuno et al., Nucl. Instr. and Meth. A, 676, 54 (2012).

[7] S. Kawana et al., Nucl. Instr. and Meth. A, 681, 68 (2012).

[8] Y. Tameda et al., 32th Proc. of International Cosmic Ray Conference, HE1.3, 1268 (2011). 
[9] D. Ikeda et al., 32th Proc. of International Cosmic Ray Conference, HE1.3, 1264 (2011).

[10] . T. Fujii et al., International Symposium on the Recent Progress of Ultra-High Energy Cosmic Ray Observation, AIP Conf. Proc. 1367, 149 (2011).

[11] D. Heck et al., Forschungszentrum Karlsruhe Report FZKA, 6019 (1998).

[12] T. Abu-Zayyad et al., Astropart. Phys., 39-40, 109 (2012)
[13] D. Ivanov et al., 32th Proc. of International Cosmic Ray Conference, HE1.3, 1297 (2011).

[14] M. Nagano, et al., J. Phys. G 18, 447 (1992).

[15] M. Takeda et al., Phys. Rev. Lett., 81(6), 1163 (1998)

[16] J. Abraham, et al., Phys. Lev. Lett. 101, 061101 (2008).

[17] Y. Tsunesada et al., 32th Proc. of International Cosmic Ray Conference, HE1.3, 1270 (2011). 